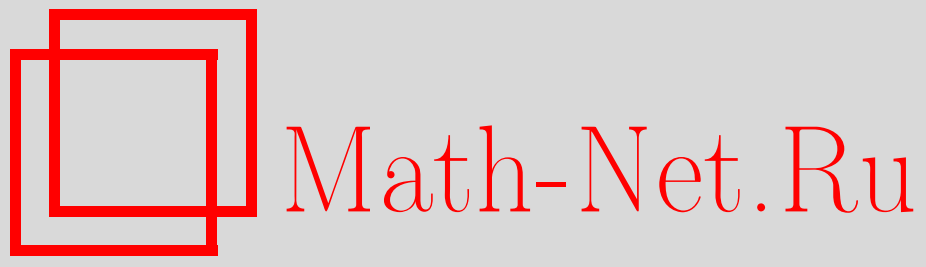

В. А. Быковский, Образующие элементы аннулирующего идеала для модулярных символов, Функи. анализ и его прил., 2003, том 37, выпуск 4, 27-38

DOI: https://doi.org/10.4213/faa166

Использование Общероссийского математического портала MathNet.Ru подразумевает, что вы прочитали и согласны с пользовательским соглашением

http://www . mathnet.ru/rus/agreement

Параметры загрузки:

IP : 54.205.225.156

26 апреля 2023 г., 17:18:12

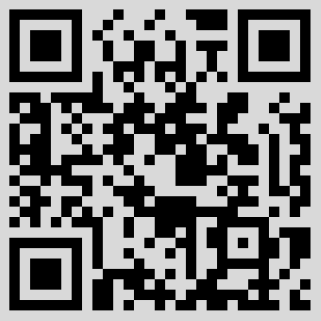


Функциональный анализ и его приложения

2003, т. 37, вып. 4, с. 27-38

УДК $512.743+512.817$

\title{
Образующие элементы аннулирующего идеала для модулярных символов*
}

\author{
(c) 2003. В. А. Быковский
}

Посвящается И. М. Гельфанду в связи с его 90-летием

\section{Введение}

Периоды модулярных форм и собственные значения операторов Гекке содержат в себе важную арифметическую информацию о специальных абелевых алгебраических многообразиях (эллиптических кривых, якобианах модулярных кривых, многообразиях Куги-Сато, ...) и неприводимых двумерных $l$-адических представлениях группы Галуа. Отображение пространств модулярных форм в периоды, построенное в основополагающей работе $M$. Эйхлера [1] и детально изученное Г. Шимурой [2], отождествляет их со специальными подпространствами в $\mathbb{R}^{n}$, выделяемыми с помощью соотношений Эйхлера-Шимуры. Дальнейшее развитие этих идей в работах Ю. И. Манина [3-5] и других авторов (см. $[6,7]$ ) привело к фундаментальному понятию «модулярный символ». Речь идет о следующей конструкции.

Пусть $\mathscr{M}_{s}(\mathbb{Z})(s=2,3, \ldots)$ состоит из всех целочисленных матриц

$$
M=\left(m^{1}, \ldots, m^{s}\right)
$$

с ненулевыми $s$-столбцами $m^{i}$. Обозначим через $\mathfrak{S}_{s}(\mathbb{Z})$ фактормодуль

$$
\mathbb{Z}\left[\mathscr{M}_{s}(\mathbb{Z})\right] / \mathbb{Z}_{0}\left[\mathscr{M}_{s}(\mathbb{Z})\right],
$$

где $\mathbb{Z}\left[\mathscr{M}_{s}(\mathbb{Z})\right]$ - свободная абелева группа, порожденная формальными образующими $M$ из $\mathscr{M}_{s}(\mathbb{Z})$, а $\mathbb{Z}_{0}\left[\mathscr{M}_{s}(\mathbb{Z})\right]-$ ее подгруппа, порожденная элементами

(1) $\left(a_{1} m^{1}, \ldots, a_{s} m^{s}\right)-\left(m^{1}, \ldots, m^{s}\right)$ с любыми целыми $a_{i} \neq 0$;

(2) $\left(m^{\sigma(1)}, \ldots, m^{\sigma(s)}\right)-\operatorname{sign}(\sigma)\left(m^{1}, \ldots, m^{s}\right)$ для всех перестановок $\sigma$;

(3) $\left(m^{1}, \ldots, m^{s}\right)$, если $m^{1}, \ldots, m^{s}$ линейно зависимы;

(4) $\sum_{i=0}^{s}(-1)^{i}\left(m^{0}, \ldots, m^{i-1}, m^{i+1}, \ldots, m^{s}\right)=\sum_{i=0}^{s}(-1)^{i}\left(m^{0}, \ldots, \widehat{m}^{i}, \ldots, m^{s}\right)$ для всех ненулевых $s$-столбцов $m^{0}, m^{1}, \ldots, m^{s}$.

При каноническом проектировании $\mathbb{Z}\left[\mathscr{M}_{s}(\mathbb{Z})\right]$ на $\mathfrak{S}_{s}(\mathbb{Z})$ элементы $M$ из $\mathscr{M}_{s}(\mathbb{Z})$ переходят в классы $[M]$ из $\mathfrak{S}_{s}(\mathbb{Z})$, именуемые модулярными символами.

ЗАмЕчАНИЕ 1. Модулярные символы можно интерпретировать как классы наборов $\left(\alpha^{1}, \ldots, \alpha^{s}\right)$ с точками $\alpha^{i}$ из $(s-1)$-мерного проективного пространства над полем рациональных чисел относительно следующей эквивалентности:

(i) $\left(\alpha^{\sigma(1)}, \ldots, \alpha^{\sigma(s)}\right) \sim \operatorname{sign}(\sigma)\left(\alpha^{1}, \ldots, \alpha^{s}\right)$;

* Работа выполнена при финансовой поддержке Российского фонда фундаментальных исследований (грант № 01-01-96301) и Президиума ДВО РАН (грант № 03-1-0-01-003). 
(ii) $\left(\alpha^{1}, \ldots, \alpha^{s}\right) \sim 0$, если $\alpha^{1}, \ldots, \alpha^{s}$ линейно зависимы;

(iii) $\sum_{i=0}^{s}(-1)\left(\alpha_{0}, \ldots, \widehat{\alpha}_{i}, \ldots, \alpha^{s}\right) \sim 0$ для любых $\alpha_{0}, \alpha_{1}, \ldots, \alpha^{s}$.

Именно в таком виде они были впервые введены Ю. И. Маниным в случае $s=2$, для которого (i) и (ii) следуют из (iii).

Левое умножение элементов групп

$$
\begin{gathered}
\bar{\Gamma}=\bar{\Gamma}_{s}=G L(s ; \mathbb{Z})=\left\{M \in \mathscr{M}_{s}(\mathbb{Z}) \mid \operatorname{det}(M)= \pm 1\right\}, \\
\Gamma=\Gamma_{s}=S L(s ; \mathbb{Z})=\{M \in \bar{\Gamma} \mid \operatorname{det}(M)=1\}
\end{gathered}
$$

на матрицы из $\mathscr{M}_{s}(\mathbb{Z})$ определяет структуры левых $\mathbb{Z}[\bar{\Gamma}]$ - и $\mathbb{Z}[\Gamma]$-модулей на $\mathbb{Z}\left[\mathscr{M}_{s}(\mathbb{Z})\right]$ и $\mathbb{Z}_{0}\left[\mathscr{M}_{s}(\mathbb{Z})\right]$, которые канонически переносятся на $\mathfrak{S}_{s}(\mathbb{Z})$. Обозначим через $E$ единичную матрицу $\left(e^{1}, \ldots, e^{s}\right)$ из $\Gamma$ с соответствующими $s$-столбцами $e^{i}, i=1, \ldots, s$. Для $s=2$ Манин [3], а для $s \geqslant 3$ Эш и Рудольф [6] установили, что

$$
\mathfrak{S}_{s}(\mathbb{Z})=\{R \cdot[E] \mid R \in \mathbb{Z}[\bar{\Gamma}]\}=\{R \cdot[E] \mid R \in \mathbb{Z}[\Gamma]\} .
$$

То есть $\mathfrak{S}_{s}(\mathbb{Z})$ есть циклический модуль над групповыми кольцами $\mathbb{Z}[\bar{\Gamma}]$ и $\mathbb{Z}[\Gamma]$ с образующей $[E]$.

Введем следующие обозначения для специальных элементов из $\bar{\Gamma}$ :

$$
\begin{gathered}
S=\left(-e^{2}, e^{1}, e^{3}, \ldots, e^{s}\right), \quad \bar{S}=\left(e^{2}, e^{1}, e^{3}, \ldots, e^{s}\right), \\
P=\left(e^{2}, e^{3}, \ldots, e^{s},(-1)^{s+1} e^{1}\right), \quad \bar{P}=\left(e^{2}, e^{3}, \ldots, e^{s}, e^{1}\right), \\
I=\left(-e^{1}, e^{2}, \ldots, e^{s}\right), \quad U=\left(-e^{1}-e^{2}, e^{1}, e^{3}, \ldots, e^{s}\right) .
\end{gathered}
$$

Отметим, что $\bar{P}=P$ для нечетных $s$.

Главным результатом работы является

Теорема 1. Левый идеал $\overline{\mathscr{L}}(s)=\{R \in \mathbb{Z}[\bar{\Gamma}] \mid R \cdot[E]=0\}$ в $\mathbb{Z}[\bar{\Gamma}]$ порождается образующими

$$
E-I, \quad E+\bar{S}, \quad E+(-1)^{s} \bar{P}, \quad E+U+U^{2} .
$$

Из нее следует

Теорема 2. Левый идеал $\mathscr{L}(s)=\{R \in \mathbb{Z}[\Gamma] \mid R \cdot[E]=0\}$ в $\mathbb{Z}[\Gamma]$ порождается образующими

$$
E+S, \quad E+(-1)^{s} P, \quad E+U+U^{2} .
$$

В сформулированных утверждениях $\mathscr{L}(s)$ и $\overline{\mathscr{L}}(s)$ есть аннулирующие идеаль в $\mathbb{Z}[\Gamma]$ и $\mathbb{Z}[\bar{\Gamma}]$ для образующей $[E]$ из $\mathfrak{S}_{s}(\mathbb{Z})$.

Пусть $L$ - произвольный левый модуль над коммутативным кольцом $K$ с единицей, на котором справа действует группа $\Gamma=S L(s ; \mathbb{Z})$. Назовем

$$
\mathscr{E}(L)=\operatorname{Hom}_{\Gamma}\left(\mathfrak{S}_{s}(\mathbb{Z}), L\right)
$$

модулем Эйхлера, а

$$
\mathscr{E}^{\prime}(L)=L \underset{\Gamma}{\otimes} \mathfrak{S}_{s}(\mathbb{Z})
$$

двойственным модулем Эйхлера. Модуль $\mathscr{E}(L)$ каноническим способом интерпретируется как левый модуль над $K$, состоящий из всех функций

$$
\Phi: \mathscr{M}_{s}(\mathbb{Z}) \rightarrow L,
$$

таких, что 
a) $\Phi\left(a_{1} m^{1}, \ldots, a_{s} m^{s}\right)=\Phi\left(m^{1}, \ldots, m^{s}\right)$ для всех целых $a_{i} \neq 0$;

b) $\Phi\left(m^{\sigma(1)}, \ldots, m^{\sigma(s)}\right)=\operatorname{sign}(\sigma) \Phi\left(m^{1}, \ldots, m^{s}\right)$ для любой перестановки $\sigma$;

c) $\Phi\left(m^{1}, \ldots, m^{s}\right)=0$, если $m^{1}, \ldots, m^{s}$ линейно зависимы;

d) для произвольных ненулевых столбцов $m^{0}, m^{1}, \ldots, m^{s}$

$$
\sum_{i=0}^{s}(-1)^{i} \Phi\left(m^{0}, \ldots, \widehat{m}^{i}, \ldots, m^{s}\right)=0 ;
$$

е) $\Phi(Q M)=\Phi(M) \circ Q^{-1}$ для любого $Q \in \Gamma$.

Для размерности $s=2$ модуль параболических коциклов Эйхлера (см. [2-4]), ассоциированный с пространством модулярных функций для подгрупп конечного индекса в $S L(2 ; \mathbb{Z})$, при соответствующем выборе модуля $L$ канонически изоморфен $\mathscr{E}(L)$. В частности, в качестве $L$ пространству $S L(2 ; \mathbb{Z})$-инвариантных модулярных форм четного положительного веса $k$ соответствует вещественное линейное пространство однородных полиномов степени $k-2$ от двух переменных, на котором стандартно действует $S L(2 ; \mathbb{Z})$ путем линейной замены последних. Именно по этой причине мы назвали $\mathscr{E}(L)$ модулем Эйхлера.

Обозначим через $\mathfrak{M}(L)$ подмодуль в $L$, состоящий из всех $A \in L$, для которых

$$
A+A \circ S=A+(-1)^{s} A \circ P=A+A \circ U+A \circ U^{2}=0,
$$

а через $\mathfrak{M}^{\prime}(L)$ фактормодуль модуля $L$ по подмодулю

$$
L_{0}=\left\{A_{1} \circ(E+S)+A_{2} \circ\left(E+(-1)^{s} P\right)+A_{3} \circ\left(E+U+U^{2}\right) \mid \forall A_{1}, A_{2}, A_{3} \in L\right\} .
$$

Мы их назовем модулями Эйхлера-Шимуры $\left(\mathfrak{M}^{\prime}(L)-\right.$ двойственный к $\left.\mathfrak{M}(L)\right)$, продолжая традицию, относящуюся к случаю $s=2$. Из теоремы 2 непосредственно следует

Теорема 3. Соответствия

$$
\Phi \rightarrow \Phi(E), \quad A \underset{\Gamma}{\otimes}[E] \rightarrow A+L_{0}
$$

определяют канонические изоморфизмы

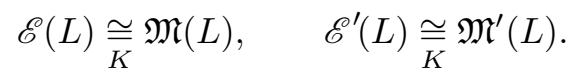

Этот результат позволяет стандартным способом построить теорию операторов Гекке в $\mathfrak{M}(L)$ и $\mathfrak{M}^{\prime}(L)$ для произвольных модулей $L$, на которых действует справа полугруппа целочисленных матриц $Q$ из $\mathscr{M}_{s}(\mathbb{Z}) \mathrm{c} \operatorname{det}(Q)>0$. Очевидно, что для конкретных конечномерных модулей $L$ модули Эйхлера-Шимуры (алгебраические аналоги пространств модулярных форм) и собственные значения операторов Гекке можно непосредственно вычислить. Этот факт имеет принципиальное значение при проведении численных экспериментов по поводу многомерной гипотезы Серра о модулярных представлениях группы Галуа (см. [8]).

Теорема 2 позволяет вычислять гомологии и когомологии

$$
H_{\nu}\left(\Gamma^{\prime}: L\right), \quad H^{\nu}\left(\Gamma^{\prime}: L\right)
$$

для подгрупп $\Gamma^{\prime}$ конечного индекса в $\Gamma$. Здесь $\nu=s(s-1) / 2-$ виртуальная когомологическая размерность группы $\Gamma$ (см. [9]). Предположим, что кручения группы Г обратимы в кольце $K$. Борель и Серр [10] доказали, что модуль Стейнберга

$$
S t_{\Gamma^{\prime}}(s)=H^{\nu}\left(\Gamma^{\prime}: \mathbb{Z}\left[\Gamma^{\prime}\right]\right)
$$


есть свободная от кручения абелева группа счетного ранга с левым действием группы $\Gamma^{\prime}$. При этом имеют место канонические изоморфизмы Бореля-Серра

$$
\begin{gathered}
H_{k}\left(\Gamma^{\prime}: L\right) \cong H^{\nu-k}\left(\Gamma^{\prime}: \operatorname{Hom}\left(S t_{\Gamma^{\prime}}(s), L\right)\right), \\
H^{k}\left(\Gamma^{\prime}: L\right) \cong H^{\nu-k}\left(\Gamma^{\prime}: L \otimes S t_{\Gamma^{\prime}}(s)\right)
\end{gathered}
$$

для $0 \leqslant k \leqslant \nu$. С помощью леммы Шапиро для $k=\nu$ их можно представить в виде

$$
\begin{gathered}
H_{\nu}\left(\Gamma^{\prime}: L\right) \underset{\bar{K}}{\cong} H^{0}\left(\Gamma: \operatorname{Hom}\left(S t(s), \mathscr{F}\left(\Gamma / \Gamma^{\prime}\right) \underset{K}{\otimes} L\right)\right), \\
H^{\nu}\left(\Gamma^{\prime}: L\right) \underset{K}{\cong} H_{0}\left(\Gamma:\left(\mathscr{F}\left(\Gamma / \Gamma^{\prime}\right) \underset{K}{\otimes} L\right) \otimes S t(s)\right),
\end{gathered}
$$

где $S t(s)=S t_{\Gamma}(s)$. Здесь $\mathscr{F}\left(\Gamma / \Gamma^{\prime}\right)$ левый модуль над $K$, состоящий из функций $F: \Gamma / \Gamma^{\prime} \rightarrow K$. Правое действие группы $\Gamma$ на $\mathscr{F}\left(\Gamma / \Gamma^{\prime}\right) \otimes L$ определяется по правилу

$$
(F \otimes A) \circ Q=(F \circ Q) \otimes(A \circ Q), \quad(F \circ Q)\left(G \Gamma^{\prime}\right)=F\left(Q G \Gamma^{\prime}\right)
$$

для всех $A$ из $L$, а также $Q$ и $G$ из $\Gamma$.

Поскольку (см. [6] и [7])

$$
K \otimes S t(s) \underset{\bar{\Gamma}}{\cong} \otimes \mathfrak{S}_{s}(\mathbb{Z})
$$

то справедлива следующая

ТЕОРема 4. Имеют место канонические изоморфизмы

$$
H_{\nu}\left(\Gamma^{\prime}: L\right) \underset{K}{\cong} \mathfrak{M}\left(\mathscr{F}\left(\Gamma / \Gamma^{\prime}\right) \underset{K}{\otimes} L\right), \quad H^{\nu}\left(\Gamma^{\prime}: L\right) \underset{K}{\cong} \mathfrak{M}^{\prime}\left(\mathscr{F}\left(\Gamma / \Gamma^{\prime}\right) \underset{K}{\otimes} L\right) .
$$

Тем самым утверждение теоремы (4.13) из [11] распространяется на остальные $s \geqslant 5$.

Теперь несколько слов по поводу истории вопроса.

Утверждение теоремы 1 при $s=2$ (в несколько ином виде) получено Маниным [3], а также Ридером (см. [12] и [13]), который отметил, что топологические методы Суле [14] и Эша [11] позволяют доказать ее и для $s=3,4$. Однако они не работают при $s>4$ по причинам, объясненным в [11].

Автор благодарен А. Эшу и М. Ридеру за обсуждение полученных результатов, а также рецензенту за ценные замечания.

\section{§1. Модулярные элементы}

Пусть $\mathscr{P}_{s}(\mathbb{Z})$ состоит из всех целочисленных $s \times s$-матриц $M=\left(m^{1}, \ldots, m^{s}\right)$ с $s$-столбцами $m^{i}$, у которых

$$
\text { НОД }\left(m_{1}^{i}, \ldots, m_{s}^{i}\right)=1
$$

(т. е. с примитивными $s$-столбцами). Назовем невырожденную матрицу $N$ из $\mathscr{P}_{s}(\mathbb{Z})$ приведенной, если она верхнетреугольная и

$$
N=\left(n_{1}^{1} e^{1}, n_{1}^{2} e^{1}+n_{2}^{2} e^{2}, \ldots, n_{1}^{i} e^{1}+\cdots+n_{i}^{i} e^{i}, \ldots, n_{1}^{s} e^{1}+\cdots+n_{s}^{s} e^{s}\right)
$$

с $0 \leqslant n_{j}^{i}<n_{i}^{i}(1 \leqslant j<i \leqslant s)$. Ее диагональные элементы положительны и всегда $n_{1}^{1}=1$. 
Из теории целочисленных решеток хорошо известно, что любую невырожденную матрицу $M \in \mathscr{P}_{s}(\mathbb{Z})$ можно представить в виде $M=Q N$ с приведенной матрицей $N \in \mathscr{P}_{s}(\mathbb{Z})$ и $Q \in \bar{\Gamma}$. Если

$$
M=Q N=Q^{\prime} N^{\prime}
$$

- два таких разложения, то

$$
N=Q^{-1} Q^{\prime} N^{\prime}=Q_{1} N^{\prime},
$$

где $Q_{1} \in \bar{\Gamma}-$ верхнетреугольная матрица. Последовательная проверка условия приведенности матриц $N$ и $N^{\prime}$ по столбцам, начиная со второго, немедленно приводит к равенству

$$
Q^{-1} Q^{\prime}=Q_{1}=E .
$$

Поэтому разложение $M=Q N$ единственно. Этот факт в дальнейшем играет принципиальную роль.

Пусть $t=t(M)$ есть наибольшее натуральное $t$, для которого $n_{i}^{i}=1$ при всех $1 \leqslant i<t$. При этом $t(M)=s+1$ только для $M$ из $\bar{\Gamma}$. В случае $t(M) \leqslant s$ положим

$$
M_{i}=Q\left(e^{t}, n^{1}, \ldots, \widehat{n}^{i}, \ldots, n^{s}\right)=\left(Q\left(e^{t}\right), m^{1}, \ldots, \widehat{m}^{i}, \ldots, m^{s}\right)
$$

(столбцы $n^{i}$ и $m^{i}$ удаляются). Это снова элементы из $\mathscr{P}_{s}(\mathbb{Z})$. Легко проверить, что

$$
\left|\operatorname{det}\left(M_{i}\right)\right|<|\operatorname{det}(M)| \quad(1 \leqslant i \leqslant s) .
$$

Зафиксируем произвольный левый $\mathbb{Z}[\bar{\Gamma}]$-модуль $\mathscr{H}$ (абелеву группу, на которой слева действует группа $\bar{\Gamma})$ и обозначим через $\mathfrak{N}(\mathscr{H})$ подгруппу в $\mathscr{H}$, состоящую из всех элементов $H$, для которых

$$
(E-I) \cdot H=(E+\bar{S}) \cdot H=\left(E+(-1)^{s} \bar{P}\right) \cdot H=\left(E+U+U^{2}\right) \cdot H=0 .
$$

ПримеР. Для левого идеала $\overline{\mathscr{L}}(s)$ в $\mathbb{Z}[\bar{\Gamma}]$ из теоремы 1 , сформулированной во введении, определим фактормодуль $\mathscr{H}=\mathbb{Z}[\bar{\Gamma}] / \overline{\mathscr{L}}(s)$. Тогда $H=E+\overline{\mathscr{L}}(s)$ есть элемент из $\mathfrak{N}(\mathscr{H})$.

Зафиксируем произвольный элемент $H$ из $\mathfrak{N}(\mathscr{H})$ и построим отображение

$$
M \rightarrow[M]_{H}
$$

из $\mathscr{P}_{s}(\mathbb{Z})$ в $\mathscr{H}$ по следующему правилу:

1) $[M]_{H}=0$, если $\operatorname{det}(M)=0$;

2) $[M]_{H}=M \cdot H$, если $M \in \bar{\Gamma}$;

3) если $|\operatorname{det}(M)|>1$, то $[M]_{H}$ определяется рекурсивно из соотношения

$$
[M]_{H}+\sum_{i=1}^{s}(-1)^{i}\left[M_{i}\right]_{H}=0
$$

в соответствии с $(1.1)$ и (1.2).

Элементы $[M]_{H}$ из $\mathscr{H}$ мы будем называть модулярными әлементами.

ЗАмЕЧАНИЕ 2. В основу этой конструкции положен алгоритм для вычисления модулярных символов через унимодулярные символы $[M]$ с $M \in \Gamma$ из [6].

Содержательная часть настоящей работы связана с доказательством следующего утверждения. 
ОсНовная теорема. Для модулярных элементов $[M]_{H}=\left[m^{1}, \ldots, m^{s}\right]_{H}$ вblполняются следующие соотношения:

(I) $[Q M]_{H}=Q[M]_{H}$ для любого $Q \in \bar{\Gamma}$;

(II) $\left[\varepsilon_{1} m^{1}, \ldots, \varepsilon_{s} m^{s}\right]_{H}=\left[m^{1}, \ldots, m^{s}\right]_{H}$ для любых $\varepsilon_{i} \in\{ \pm 1\}$;

(III) для любой перестановки $\sigma$

$$
\left[m^{\sigma(1)}, \ldots, m^{\sigma(s)}\right]_{H}=\operatorname{sign}(\sigma)\left[m^{1}, \ldots, m^{s}\right]_{H} ;
$$

(IV) ecлu $\operatorname{det}(M)=0$, mo $[M]_{H}=0$;

(V) для любых s-столбизов $m^{0}, m^{1}, \ldots, m^{s}$

$$
\sum_{i=0}^{s}(-1)^{s}\left[m^{0}, \ldots, \widehat{m}^{i}, \ldots, m^{s}\right]_{H}=0 .
$$

Эту теорему мы докажем позднее. А сейчас покажем, как из нее выводятся теоремы 1 и 2, сформулированные во введении. В соответствии с замечанием 1 из введения мы можем рассматривать модулярные символы $[M]$ только для матриц $M$ из $\mathscr{P}_{s}(\mathbb{Z})$.

Применяя основную теорему с $H=E+\overline{\mathscr{L}}(s)$, получаем, что соответствие

$$
R \cdot(E+\overline{\mathscr{L}}(s)) \rightarrow R[E] \quad \forall R \in \mathbb{Z}[\bar{\Gamma}]
$$

определяет изоморфизм между $\mathbb{Z}[\bar{\Gamma}] / \overline{\mathscr{L}}(s)$ и $\mathfrak{S}_{s}(\mathbb{Z})$, что и утверждается в теореме 1 . Каждый элемент $R \in \mathbb{Z}[\bar{\Gamma}]$ однозначно представляется в виде $R=R_{0}+R_{1} I$ с $R_{0}$ и $R_{1}$ из $\mathbb{Z}[\Gamma]$. При этом $I \cdot \mathbb{Z}[\Gamma]=\mathbb{Z}[\Gamma] \cdot I$. Кроме того,

$$
\begin{gathered}
E+\bar{S}=(E+S)-S(E-I), \\
I(E+\bar{S})=\left(E-S+S^{2}\right)(E+S)-(E-I), \\
E+(-1)^{s} \bar{P}=(E-S)(E+S)+S^{2}\left(E+(-1)^{s} P\right)-(-1)^{s} S^{2} P(E-I), \\
I\left(E+(-1)^{s} \bar{P}\right)=\left(E+(-1)^{s} P\right)-(E-I), \\
I=S E S^{-1} I, \quad I U=S U^{2} S^{-1} I, \quad I U^{2}=S U S^{-1} I .
\end{gathered}
$$

Отсюда следует, что элементы $R$ из $\mathbb{Z}[\Gamma]$, принадлежащие идеалу $\overline{\mathscr{L}}(s)$, составляют идеал $\mathscr{L}(s)$ в $\mathbb{Z}[\Gamma]$, определенный во введении. А это и есть утверждение теоремы 2 .

\section{§2. Вспомогательные утверждения}

Положим по определению

$$
\delta_{H}\left(m^{0}, m^{1}, \ldots, m^{s}\right)=\sum_{i=0}^{s}(-1)^{i}\left[m^{0}, \ldots, \widehat{m}^{i}, \ldots, m^{s}\right]_{H} .
$$

В п. (V) основной теоремы утверждается, что это выражение всегда равно нулю. Нашим главным инструментом при его доказательстве будет следующее очевидное утверждение (аналог равенства $d d(\ldots)=0$ из гомологической алгебры).

Лемма 1. Пусть $m, m^{0}, m^{1}, \ldots, m^{s}-$ некоторые примитивные $s$-столбцьь, для которых

$$
\delta_{H}\left(m, m^{0}, \ldots, \widehat{m}^{i}, \ldots, m^{s}\right)=0 \quad(i=0,1, \ldots, s) .
$$


Тогда $\delta_{H}\left(m^{0}, m^{1}, \ldots, m^{s}\right)=0$.

Действуя на $\delta_{H}(\ldots)$ слева матрицами из $\bar{\Gamma}$, получим еще одно полезное утверждение.

ЛЕмма 2. Пусть $\delta_{H}\left(m^{0}, m^{1}, \ldots, m^{s}\right)=0$. Тогда $\delta_{H}\left(n^{0}, n^{1}, \ldots, n^{s}\right)=0$ для любой матриць $\left(n^{0}, n^{1}, \ldots, n^{s}\right)$, которая получается из $\left(m^{0}, m^{1}, \ldots, m^{s}\right)$ nерестановкой строк, изменением у некоторых из них знака, а также добавлениями к любой строке целочисленной линейной комбинации остальных строк.

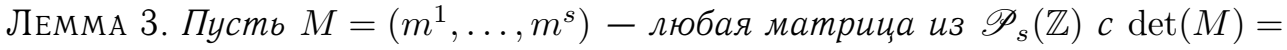
0, \pm 1 . Тогда

(a) $\left[\varepsilon_{1} m^{1}, \ldots, \varepsilon_{s} m^{s}\right]_{H}=\left[m^{1}, \ldots, m^{s}\right]_{H}$ для любых $\varepsilon_{i} \in\{ \pm 1\}$;

(b) для любой перестановки б

$$
\left[m^{\sigma(1)}, \ldots, m^{\sigma(s)}\right]_{H}=\operatorname{sign}(\sigma)\left[m^{1}, \ldots, m^{s}\right]_{H} .
$$

ДоКАЗАТЕЛьство. Утверждение тривиально для $\operatorname{det}(M)=0$. Пусть теперь $M=Q \in \bar{\Gamma}$. Так как $H \in \mathfrak{N}(\mathscr{H})$, то

$$
[I]_{H}=[E]_{H}, \quad[\bar{S}]_{H}=-[E]_{H}, \quad[\bar{P}]_{H}=(-1)^{s}[E]_{H} .
$$

Элементы $\bar{S}$ и $\bar{P}$ порождают конечную мультипликативную группу $\{E(\sigma)=$ $\left(e^{\sigma(1)}, \ldots, e^{\sigma(s)}\right) \mid$ все перестановки $\left.\sigma\right\}$. Поэтому $[E(\sigma)]_{H}=\operatorname{sign}(\sigma)[E]_{H}$ и

$$
\begin{aligned}
{\left[\varepsilon_{1} m^{\sigma(1)}, \ldots, \varepsilon_{s} m^{\sigma(s)}\right]_{H} } & =\left[Q \left(\varepsilon_{1} e^{\sigma(1)}, \ldots, Q\left(\varepsilon_{s} e^{\sigma(s)}\right]_{H}=Q E(\sigma) \cdot\left[\varepsilon_{1} e^{1}, \ldots, \varepsilon_{s} e^{s}\right]_{H}\right.\right. \\
& =Q E(\sigma) I^{\varepsilon_{1}}\left(\bar{P}^{-1} I^{\varepsilon_{2}} \bar{P}^{1}\right) \ldots\left(\bar{P}^{-(s-1)} I^{\varepsilon_{s}} \bar{P}^{s-1}\right) \cdot[E]_{H} \\
& =Q E(\sigma) \cdot[E]_{H}=\operatorname{sign}(\sigma)[Q]_{H}=\operatorname{sign}(\sigma)\left[m^{1}, \ldots, m^{s}\right]_{H} .
\end{aligned}
$$

Лемма 3 доказана.

ЛЕмма 4. Пусть $M=Q N-$ невьрожденная матрицуа из $\mathscr{P}_{s}(\mathbb{Z})$, где $Q \in$ $\bar{\Gamma}$ и $N$ - приведенная матрица с $n_{1}^{1}=\cdots=n_{s-1}^{s-1}=1$. Тогда для любой перестановки $\sigma$ с $\sigma(s)=s$

$$
\left[m^{\sigma(1)}, \ldots, m^{\sigma(s-1)}, m^{s}\right]_{H}=\operatorname{sign}(\sigma)\left[m^{1}, \ldots, m^{s-1}, m^{s}\right]_{H} .
$$

ДокАЗАТЕльство. Для $n_{s}^{s}=1$ утверждение следует из леммы 3. Далее проводим индукцию по $n_{s}^{s}$ с помощью (1.4).

Следующее утверждение - одно из ключевых мест в работе.

ЛЕммА 5. Пусть $m^{0}, m^{1}, \ldots, m^{s}-$ примитивные $s$-столбциь $c$

$$
\operatorname{det}\left(m^{0}, \ldots, \widehat{m}^{i}, \ldots, m^{s}\right) \in\{0, \pm 1\} \quad(i=0,1, \ldots, s) .
$$

Тогда $\delta_{H}\left(m^{0}, \ldots, m^{s}\right)=0$.

ДокАЗАТЕЛьство. Если $\operatorname{det}\left(m^{0}, \ldots, \widehat{m}^{i}, \ldots, m^{s}\right)=0$ при всех $i=0,1 \ldots, s$, то утверждение тривиально. Предположим теперь, что это не так и $n+1$ есть число номеров $i$, для которых $\operatorname{det}\left(m^{0}, \ldots, \widehat{m}^{i}, \ldots, m^{s}\right)= \pm 1$. Докажем нужное равенство индукцией по $n$. Принимая во внимание лемму 3 , мы можем считать, что $Q=\left(m^{1}, \ldots, m^{s}\right) \in \bar{\Gamma}$. После умножения на $Q^{-1}$ слева доказываемое равенство приобретает вид $\delta_{H}\left(Q^{-1} \cdot m^{0}, e^{1}, \ldots, e^{s}\right)=0$. При этом компоненты столбца 
$Q^{-1} \cdot m^{0}$ принимают значения $0, \pm 1$. Последнее равенство с помощью леммы 3 преобразуется к виду

$$
\delta_{H}\left(e^{1}+\cdots+e^{n}, e^{1}, \ldots, e^{s}\right)=0 .
$$

При $n=1$ оно тривиально, а в случае $n=2$ речь идет о соотношении

$$
\left[e^{1}, \ldots, e^{s}\right]_{H}-\left[e^{1}+e^{2}, \hat{e}^{1}, e^{2}, \ldots, e^{s}\right]_{H}+\left[e^{1}+e^{2}, e^{1}, \hat{e}^{2}, \ldots, e^{s}\right]_{H}=0 .
$$

Оно также выполнено, поскольку (см. лемму 3 )

$$
\begin{aligned}
{\left[e^{1}+e^{2}, e^{1}, \hat{e}^{2}, \ldots, e^{s}\right]_{H} } & =\left[-e^{1}-e^{2}, e^{1}, e^{3}, \ldots, e^{s}\right]_{H}=U \cdot[E]_{H}, \\
-\left[e^{1}+e^{2}, \hat{e}^{1}, e^{2}, \ldots, e^{s}\right]_{H} & =\left[e^{2},-e^{1}-e^{2}, e^{3}, \ldots, e^{s}\right]_{H}=U^{2} \cdot[E]_{H}
\end{aligned}
$$

и по условию (см. (1.3))

$$
[E]_{H}+U \cdot[E]_{H}+U^{2} \cdot[E]_{H}=H+U \cdot H+U^{2} \cdot H=0 .
$$

Пусть теперь $n \geqslant 3$. Положим в лемме 1

$$
m=e^{1}+\cdots+e^{n-1}, \quad m^{0}=e^{1}+\cdots+e^{n}, \quad m^{i}=e^{i} \quad(i=1, \ldots, s) .
$$

Заметим, что (предположение индукции)

$$
\delta_{H}\left(m, m^{1}, \ldots, m^{s}\right)=0,
$$

поскольку среди матриц

$$
\left(e^{1}+\cdots+e^{n-1}, e^{1}, \ldots, \hat{e}^{j}, \ldots, e^{s}\right)
$$

ровно $n-1$ невырожденных для $1 \leqslant j<n$. По той же причине

$$
\begin{array}{cl}
\delta_{H}\left(m, m^{0}, \ldots, \widehat{m}^{j}, \ldots, m^{s}\right)=0 & (1 \leqslant j<n), \\
\delta_{H}\left(m, m^{0}, \ldots, \widehat{m}^{n}, \ldots, m^{s}\right)=0, & (n<j \leqslant s) .
\end{array}
$$

В первом случае среди матриц, получаемых удалением столбца из

$$
\left(e^{1}+\cdots+e^{n-1}, e^{1}+\cdots+e^{n}, e^{1}, \ldots, e^{j-1}, e^{j+1}, \ldots e^{n} \ldots, e^{s}\right),
$$

только три невырожденные (удаляются первый или второй столбцы или $e^{n}$ ). Во втором случае только после удаления одного из $n-1$ столбцов $e^{1}, \ldots, e^{n-1}$ или первого столбца из набора

$$
\left(e^{1}+\cdots+e^{n-1}, e^{1}+\cdots+e^{n}, e^{1}, \ldots, e^{n-1}, e^{n+1}, \ldots, e^{s}\right)
$$

получаются невырожденные матрицы (всего $n$ ). В третьем случае после удаления любого столбца из

$$
\left(e^{1}+\cdots+e^{n-1}, e^{1}+\cdots+e^{n}, e^{1}, \ldots, e^{n}, \ldots, e^{j-1}, e^{j+1}, \ldots, e^{s}\right)
$$

получаются только вырожденные матрицы. Лемма 5 полностью доказана.

ЛЕмма 6. Пусть $m=m_{1} e^{1}+\cdots+m_{s} e^{s}-$ nроизвольный примитивный s-столбец с неотрицательными $m_{i}$, среди которых только одно максимальное. Тогда $\delta_{H}\left(e^{1}, \ldots, e^{s}, m\right)=0$.

ДокАЗАТЕЛЬСтво. В случае максимального $m_{s}$ утверждение непосредственно следует из (1.4) и леммы 4. Применяя лемму 2 и лемму 4, распространяем его на общий случай. 
Лемма 7. Пусть m есть любой примитивный $s$-столбец. Тогда $\delta_{H}\left(e^{1}, \ldots\right.$, $\left.e^{s}, m\right)=0$.

ДокАЗАТЕЛЬСтво. Пусть $|m|_{\infty}=\max \left\{\left|m_{1}\right|, \ldots,\left|m_{s}\right|\right\}$. Для $|m|_{\infty}=1$ утверждение непосредственно следует из леммы 5 . Пусть оно справедливо для всех $m$ с $|m|_{\infty}<q$, где $q>1$. Предположим теперь, что $|m|_{\infty}=q$. Умножив доказываемое равенство на подходящую матрицу $Q \in \bar{\Gamma}$, представим его в виде (см. также лемму 4)

$$
\delta_{H}\left(e^{1}, \ldots, e^{s}, m_{1} e^{1}+\cdots+m_{t} e^{t}\right)=0,
$$

где $m_{i} \neq 0(i=1, \ldots, t)$. Положим $\varepsilon_{i}=\operatorname{sign}\left(m_{i}\right)$. Из леммы 5 следует, что $\delta_{H}\left(\varepsilon_{1} e^{1}+\cdots+\varepsilon_{t} e^{t}, e^{1}, \ldots, e^{s}\right)=0$. Для $t<i \leqslant s$

$$
\delta_{H}\left(\varepsilon_{1} e^{1}+\cdots+\varepsilon_{t} e^{t}, e^{1}, \ldots, \hat{e}^{i}, \ldots, e^{s}, m\right)=0,
$$

поскольку все $i$-компоненты из рассматриваемого набора $s$-столбцов равны нулю. Пусть теперь $1 \leqslant i<t$. В этом случае после умножения слева на подходящую матрицу из $\bar{\Gamma}$ равенство (2.3) (пока предполагаемое) преобразуется к виду

$$
\delta_{H}\left(e^{1} \ldots, e^{s}, m^{i}\right)=0, \quad m^{i}=\left|m^{i}\right| e^{i}+\sum_{\substack{1 \leqslant j<t \\ j \neq i}} \varepsilon_{j}\left(\left|m_{j}\right|-\left|m_{i}\right|\right) e^{j} .
$$

При этом $\left|m^{i}\right|_{\infty} \leqslant|m|_{\infty}$. Принимая во внимание лемму 1 и предположение индукции, отсюда заключаем, что равенство $(2.2)$ достаточно доказать для случая $m_{t}=q,\left|m_{i}\right|<q(1 \leqslant i<t)$. Для неотрицательных $m_{1}, \ldots, m_{t-1}$ оно следует из леммы 6 . В оставшихся случаях мы можем считать, что

$$
m=-\sum_{1 \leqslant i \leqslant l} m_{i} e^{i}+\sum_{l<i \leqslant t} m_{i} e^{i}
$$

с $1 \leqslant l<t$ и положительными $m_{i}$. Пусть

$$
\widetilde{m}=\sum_{1 \leqslant i \leqslant l}\left(q-m_{i}\right) e^{i}+\sum_{l<i \leqslant t} m_{i} e^{i} .
$$

Прибавив к каждой из первых $l$ строк строку с номером $t$ (лемма 2 ) и переставив столбец с номером $t$ на первое место (лемма 4), преобразуем (2.4) к виду

$$
\delta_{H}\left(e^{1}+\cdots+e^{l}+e^{t}, e^{1}, \ldots, \hat{e}^{t}, \ldots, e^{s}, \widetilde{m}\right)=0 .
$$

Это равенство следует из соотношений (в лемме 1 полагаем $m=e^{t}$ )

$$
\begin{gathered}
\delta_{H}\left(e^{1}+\cdots+e^{l}+e^{t}, e^{1}, \ldots, e^{s}\right)=0, \quad \delta_{H}\left(e^{1}, \ldots, e^{s}, \widetilde{m}\right)=0, \\
\delta_{H}\left(e^{1}+\cdots+e^{l}+e^{t}, e^{1}, \ldots, \hat{e}^{i}, \ldots, e^{s}, \widetilde{m}\right)=0 \quad(i \neq t) .
\end{gathered}
$$

Первое вытекает из леммы 5 , а второе из леммы 6 . Осталось проверить третью серию соотношений (2.5). Если $t<i \leqslant s$, то оно тривиально, поскольку $i$-е компоненты соответствующих $s$-столбцов равны нулю. Пусть теперь $l<i<t$. Вычитая из строки с номером $t i$-ю строку (лемма 2), заменим в $(2.5) \widetilde{m}_{t}=m_{t}=q$ на $q-m_{i}$. Применяя лемму 1 (с $e^{i}$ в качестве вспомогательного $m$ ), получим $(2.5)$ с помощью предположения индукции. Осталось рассмотреть случай $1 \leqslant i \leqslant l$. Он также сводится к предположению индукции вычитанием $i$-й строки из каждой из остальных первых $l$ строк и строки с номером $t$. Таким образом, равенство $(2.5)$, а вместе с ним (2.4) и (2.3) выполнены. Тем самым лемма 7 полностью доказана. 
ЛЕмма 8. Для любого примитивного $s$-столбия $m$ выполняется равенство

$$
\left[-e^{1}, e^{2}, \ldots, e^{s-1}, m\right]_{H}=\left[e^{1}, e^{2}, \ldots, e^{s-1}, m\right]_{H} .
$$

ДокАЗАТЕЛЬСтво. Утверждение тривиально для $m_{s}=0$. В остальных случаях с помощью леммы 2 оно приводится к случаю, когда $\left|m_{i}\right|<\left|m_{s}\right|(1 \leqslant i<s)$. Проводя индукцию по $\left|m_{s}\right|$ (см. также лемму 5), получим

$$
\begin{aligned}
{\left[-e^{1}, e^{2}, \ldots, e^{s-1}, m\right]_{H} } & =I \cdot\left[e^{1}, e^{2}, \ldots, e^{s-1}, I(m)\right]_{H} \\
& =\sum_{i=1}^{s-1}(-1)^{i-1} I \cdot\left[e^{s}, e^{1}, \ldots, \hat{e}^{i}, \ldots, e^{s-1}, I(m)\right]_{H} \\
& =\sum_{i=1}^{s-1}(-1)^{s-1} I \cdot\left[e^{s}, e^{1}, \ldots, e^{s-1}\right]_{H} \\
& =\left[e^{s}, e^{1}, \ldots, e^{i}, \ldots, e^{s-1}, m\right]_{H} \\
& \left.=e^{s-1}, m\right]_{H} .
\end{aligned}
$$

Лемма 8 доказана.

Из леммы 1 (с вспомогательным столбцом $e^{s}$ ) и леммы 5 непосредственно следует

ЛЕмма 9. При $s \geqslant 3$ для любого примитивного $s$-столбияа $m$ выполняется равенство

$$
\begin{aligned}
{\left[e^{1}, e^{2}, \ldots, e^{s-1}, m\right]_{H} } & -\left[-e^{1}-e^{2}, \hat{e}^{1}, e^{2}, \ldots, e^{s-1}, m\right]_{H} \\
+ & {\left[-e^{1}-e^{2}, e^{1}, \hat{e}^{2}, \ldots, e^{s-1}, m\right]_{H}=0 . }
\end{aligned}
$$

\section{§3. Доказательство основной теоремы}

Заметим, что пп. (I) и (IV) непосредственно следуют из определений. Для доказательства п. (V) проведем индукцию по $s$.

База индукции $(s=2)$. Речь идет о соотношении

$$
\delta_{H}\left(m^{0}, m^{1}, m^{2}\right)=\left[m^{1}, m^{2}\right]_{H}-\left[m^{0}, m^{2}\right]_{H}+\left[m^{0}, m^{1}\right]_{H}=0
$$

с любыми примитивными 2-столбцами $m^{0}, m^{1}, m^{2}$. Если $\operatorname{det}\left(m^{0}, m^{1}\right)=0$, то $m^{1}=\varepsilon m^{0}$ с $\varepsilon= \pm 1$ и равенство (3.1) приобретает вид $\left[\varepsilon m^{0}, m^{2}\right]_{H}=\left[m^{0}, m^{2}\right]_{H}$. Для $\operatorname{det}\left(m^{0}, m^{2}\right)=0$ оно тривиально, а в остальных случаях непосредственно следует из леммы 8. Выбрав подходящим образом 2 -столбец $m^{\prime}$ и матрицу $Q \in \bar{\Gamma}$ (для $s=2$ ), получим (3.1) из соотношения (лемма 7 )

$$
\left[e^{2}, m^{\prime}\right]_{H}-\left[e^{1}, m^{\prime}\right]_{H}+\left[e^{1}, e^{2}\right]_{H}=0
$$

для случая $\operatorname{det}\left(m^{0}, m^{1}\right)= \pm 1$. Далее с помощью леммы 1 и соотношения (1.4) проводим индукцию по $\left|\operatorname{det}\left(m^{0}, m^{1}\right)\right|$.

Переход от $s-1 \kappa s$. Пусть $\operatorname{det}\left(m^{0}, \ldots, m^{s-1}\right)=0$. Тогда для некоторой матрицы $Q$ из $\bar{\Gamma}$

$$
\left(m^{0}, \ldots, m^{s-1}\right)=Q\left(\bar{n}^{0}, \ldots, \bar{n}^{s-1}\right)
$$


где у $\left(\bar{n}^{0}, \ldots, \bar{n}^{s-1}\right)$ последняя строка состоит из одних нулей. Удалив ее, получим примитивные $(s-1)$-столбцы $n^{i}$. Для любого примитивного $s$-столбца $m$ обозначим через $\mathscr{H}_{m}(H) \mathbb{Z}$-подмодуль в $\mathscr{H}$, порожденный всеми элементами

$$
[N]_{H}^{m}=\left[n^{1}, \ldots, n^{s-1}\right]_{H}^{m}=\left[\bar{n}^{1}, \ldots, \bar{n}^{s-1}, m\right]_{H}=[\bar{N}, m]_{H}
$$

с $N=\left(n^{1}, \ldots, n^{s-1}\right)$ из $\mathscr{P}_{s-1}(\mathbb{Z})(N$ никак не связано с ранее фигурировавшими приведенными $s \times s$-матрицами $N)$. На $\mathscr{H}_{m}(H)$ определим левое действие группы $G L(s-1 ; \mathbb{Z})$ по правилу

$$
Q \cdot[N]_{H}^{m}=[Q N]_{H}^{m}=[\overline{Q N}, m]_{H} \cdot
$$

Из лемм 4, 8, 9 следует, что для матриц $E^{\prime}, I^{\prime}, \bar{S}^{\prime}, \bar{P}^{\prime}, U^{\prime}$ из $G L(s-1 ; \mathbb{Z})$ (они соответствуют матрицам $E, \ldots, U$ из $G L(s ; \mathbb{Z})=\bar{\Gamma})$ выполняются соотношения

$$
\begin{aligned}
\left(E^{\prime}-I^{\prime}\right) \cdot\left[E^{\prime}\right]_{H}^{m}=0, & \left(E^{\prime}+S^{\prime}\right) \cdot\left[E^{\prime}\right]_{H}^{m}=0, \\
\left(E^{\prime}+(-1)^{s-1} \bar{P}^{\prime}\right) \cdot\left[E^{\prime}\right]_{H}^{m}=0, & \left(E^{\prime}+U^{\prime}+\left(U^{\prime}\right)^{2}\right) \cdot\left[E^{\prime}\right]_{H}^{m}=0 .
\end{aligned}
$$

Принимая во внимание предположение индукции, отсюда получаем равенство

$$
\sum_{i=0}^{s-1}(-1)^{i}\left[\bar{n}^{0}, \ldots, \widehat{\bar{n}}^{i}, \ldots, \bar{n}^{s-1}, m\right]_{H}=\sum_{i=0}^{s-1}(-1)^{i}\left[n^{0}, \ldots, \widehat{n}^{i}, \ldots, n^{s-1}\right]_{H}^{m}=0 .
$$

Умножив его на $Q$ из (3.2), приходим к утверждению основной теоремы для $\operatorname{det}\left(m^{0}, \ldots, m^{s-1}\right)=0$. В случае $\operatorname{det}\left(m^{0}, \ldots, m^{s-1}\right)= \pm 1$ оно непосредственно следует из леммы 7. Проведя еще одну индукцию по $\left|\operatorname{det}\left(m^{0}, \ldots, m^{s-1}\right)\right|$ (точно так же, как при $s=2)$, с помощью (1.4) и леммы 1 получим нужное утверждение в остальных случаях. Таким образом, равенство (V) полностью доказано. Утверждения (II) и (III) легко из него получаются индукцией по $\left|\operatorname{det}\left(m^{1}, \ldots, m^{s}\right)\right|$. Основная теорема полностью доказана.

\section{ЛИТЕРАТУРА}

1. Eichler M. Eine Verallgemeinerung der Abelschen Integrale. Math. Z., 67, 267-298 (1957).

2. Shimura $G$. Introduction to the arithmetic theory of automorphic functions. Princeton Univ. Press. NJ, 1971. [Имеется перевод: Шимура Г. Введение в арифметическую теорию автоморфных функций. Мир, М., 1973.]

3. Манин Ю. И. Параболические формы и дзета-функции модулярных кривых. Изв. АН СССР, сер. матем., 36, 19-66 (1972).

4. Манин Ю. И. Периоды параболических форм и $p$-адические ряды Гекке. Матем. сб., 92, № 3, 378-401 (1973).

5. Manin Yu. I. Explicit formulas for the eigenvalues of Hecke operators. Acta Arith., 24, 239-249 (1973).

6. Ash A., Rudolph L. The modular symbol and continued fractions in higher dimensions. Invent. Math., 55, 241-250 (1979).

7. Ash A. Unstable cohomology of $S L(n, \mathscr{O})$. J. Algebra, 167, No. 2, 330-342 (1994).

8. Ash A., Sinnott $W$. An analogue of Serre's conjecture for Galois representations and Hecke eigenclasses in the $\bmod p$ cohomology of $G L(n, \mathbb{Z})$. Duke Math. J., 105, No. 2, $1-24$ (2000).

9. Brown K. Cohomology of Groups. Springer-Verlag, New York-Berlin, 1982. [Имеется перевод: Браун K. Когомологии групп. Наука, M., 1987.] 
10. Borel A., Serre J.-P. Corners and arithmetic groups. Comm. Math. Helv., 48, 436-491 (1973).

11. Ash A. Cohomology of congruence subgroups of $S L(n, \mathbb{Z})$. Math. Ann., 249, No. 1, 55-73 (1980).

12. Reeder M. Modular Symbols and Steinberg representation. Preprint. 1990

13. Reeder M. The Steinberg Module and the Cohomology of Arithmetic Groups. J. Algebra, 141, No. 2, 287-315 (1991).

14. Soule $C$. The cohomology of $S L_{3}(\mathbb{Z})$. Topology, 17, 1-22 (1978).

Хабаровское отделение Института прикладной математики Дальневосточного отделения Российской академии наук

Поступило в редакцию e-mail: vab@iam.khv.ru

13 мая 2002 г. 